\title{
Transcriptional Silencing of the TFPI-2 Gene by Promoter Hypermethylation in Choriocarcinoma Cells
}

\author{
Florent Hubé1, Pascale Reverdiau1,*, \\ Sophie lochmann', Jérôme Rollin', \\ Catherine Cherpi-Antar ${ }^{2}$ and Yves Gruel ${ }^{1}$ \\ ${ }^{1}$ Laboratoire d'Hémostase, EA 3249 'Cellules \\ Hématopoïétiques, Hémostase et Greffe', \\ ${ }^{2}$ Laboratoire de Biochimie et de Biologie Moléculaire, \\ INSERM U316, Faculté de Médecine, \\ IFR 120 'Imagerie et Exploration fonctionnelles', 2bis \\ Boulevard Tonnellé, F-37032 Tours cedex, France \\ ${ }^{*}$ Corresponding author
}

Tissue factor pathway inhibitor-2 (TFPI-2), a Kunitztype serine proteinase inhibitor associated with the extracellular matrix, has been shown to reduce tumor invasion. In the present study we identified the presence of a complete $\mathrm{CpG}$ island region spanning exon 1 and the three transcription initiation sites. We demonstrate that DNA demethylation by 5'-aza-2'-deoxycytidine restores TFPI-2 transcription in JAR choriocarcinoma cells. The effect of in vitro DNA methylation on TFPI-2 promoter function was also confirmed with TFPI-2/luciferase promoter constructs. Finally, we determined the precise methylation status of CpG sites of the TFPI-2 promoter in normal and tumor trophoblast cells using the bisulfite genomic sequencing method. We conclude that hypermethylation of the TFPI-2 gene is correlated with transcriptional silencing and that the TFPI-2 gene may be a candidate tumor suppressor gene.

Key words: 5'-Aza-2'-deoxycytidine/JAR cells / Methylation status/Normal trophoblast cells/Tissue factor pathway inhibitor 2 / Transcriptional silencing.

The degradation of the extracellular matrix (ECM) is an important step in tumor invasion and metastasis which involves a variety of proteases, particularly metalloproteinases (MMPs). Tissue factor pathway inhibitor-2 (TFPI-2), a 32 kDa Kunitz-type serine proteinase inhibitor associated with the ECM, was recently shown to reduce tumor invasion. By inhibiting plasmin and trypsin, TFPI-2 effectively decreases activation of MMP-1, MMP-3, MMP-9 and MMP-13 (Rao et al., 1999; Herman et al., 2001), thus reducing the invasive potential of several tumor cell lines (Rao et al., 1998, 2001; Konduri et al., 2000, 2001). The promoter of the human TFPI-2 gene, which is located on chromosome 7q22 (Miyagi et al., 1996), has been characterized, and we identified a complete $\mathrm{CpG}$ island region of at least $220 \mathrm{bp}$ that spans exon 1 and the three transcription initiation sites. This region, with a $\mathrm{G}+\mathrm{C}$ content of about $77 \%$ and an observed/expected presence of $\mathrm{CpG}>0.85$, also coincides with the 5 '-end of the minimal promoter. We therefore hypothesized that the TFPI-2 gene promoter could be regulated by DNA methylation and that the TFPI-2 gene might be a candidate tumor suppressor gene. CpG islands are protected from methylation in normal cells by mechanisms that are poorly understood. In contrast, hypermethylation of $\mathrm{CpG}$ islands in the promoter region of tumor suppressor genes is a known mechanism of gene silencing that contributes to tumor progression. We have reported recently that TFPI-2 transcripts are synthesized in large amounts in normal trophoblast cells isolated from term placentas (Hubé et al., 2003a), whereas they were not detected in invasive human JAR choriocarcinoma cells (Jin et al., 2001; lochmann et al., 2002) either stimulated by phorbol myristate acetate (PMA) or left unstimulated. In the present study we investigated whether the methylation status of the human TFPI-2 gene promoter could regulate the level of expression of TFPI-2 in trophoblast cells. We therefore tested the effects of 5'-aza-2'-deoxycytidine (5-aza) on the reactivation of TFPI-2 transcription in JAR cells following DNA demethylation. The effects of in vitro DNA methylation on TFPI-2 promoter function were also confirmed with TFPI-2/luciferase promoter constructs (TFPI-2/Luc). Finally, we determined the methylation status of the human TFPI-2 gene promoter in normal trophoblast cells and JAR choriocarcinoma cells using the bisulfite genomic sequencing method.

TFPI-2 mRNA synthesis was studied in normal trophoblast and JAR choriocarcinoma cells using a specific RT-PCR method. PMA, an inducer of TFPI-2 expression, was used at optimal concentration $(100 \mathrm{ng} / \mathrm{ml})$ and incubation time (4 h) as previously defined (lochmann et al., 2002). The RT-PCR product obtained appeared as a single band of $253 \mathrm{pb}$, the expected size of the TFPI-2 cDNA product, and sequencing yielding a result similar to the previously published sequence (Sprecher et al., 1994) confirmed the specificity of the technique. Expression of GAPDH used as a control was not affected in any experiment.

A large amount of TFPI-2 mRNA was detected in normal trophoblast cells, and the levels increased after stimulation with PMA (Figure 1A). In contrast, TFPI-2 mRNA 

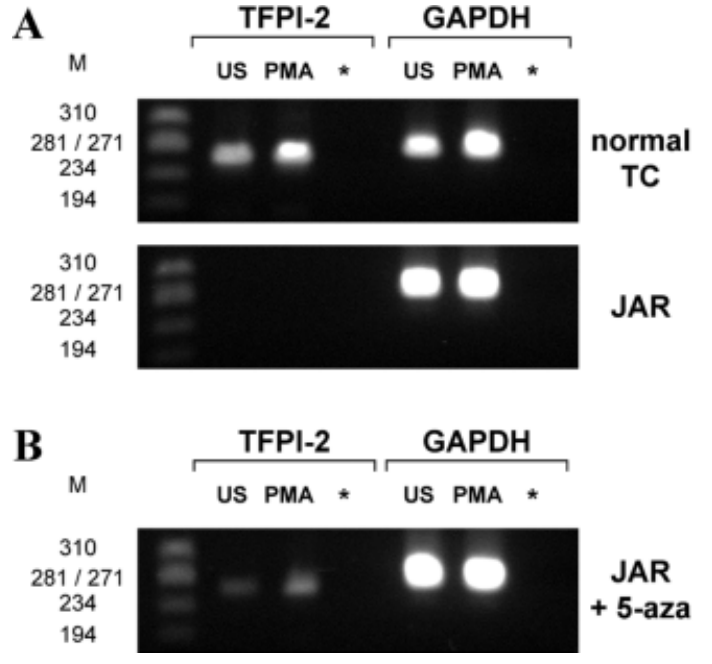

Fig. 1 TFPI-2- and GAPDH-Specific RT-PCR in Normal Trophoblast cells (TC), Choriocarcinoma Cells (JAR) and JAR Cells Treated with 5'-Aza-2'-Deoxycytidine (JAR + 5-aza).

Term placentas were supplied following spontaneous vaginal delivery in uncomplicated pregnancies after obtaining informed consent. Trophoblast cells were isolated (Hubé et al., 2003a) and grown in Waymouth/Ham's F12 medium (v/v) supplemented

A

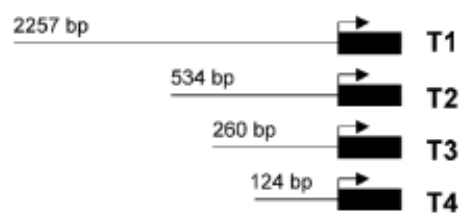

B
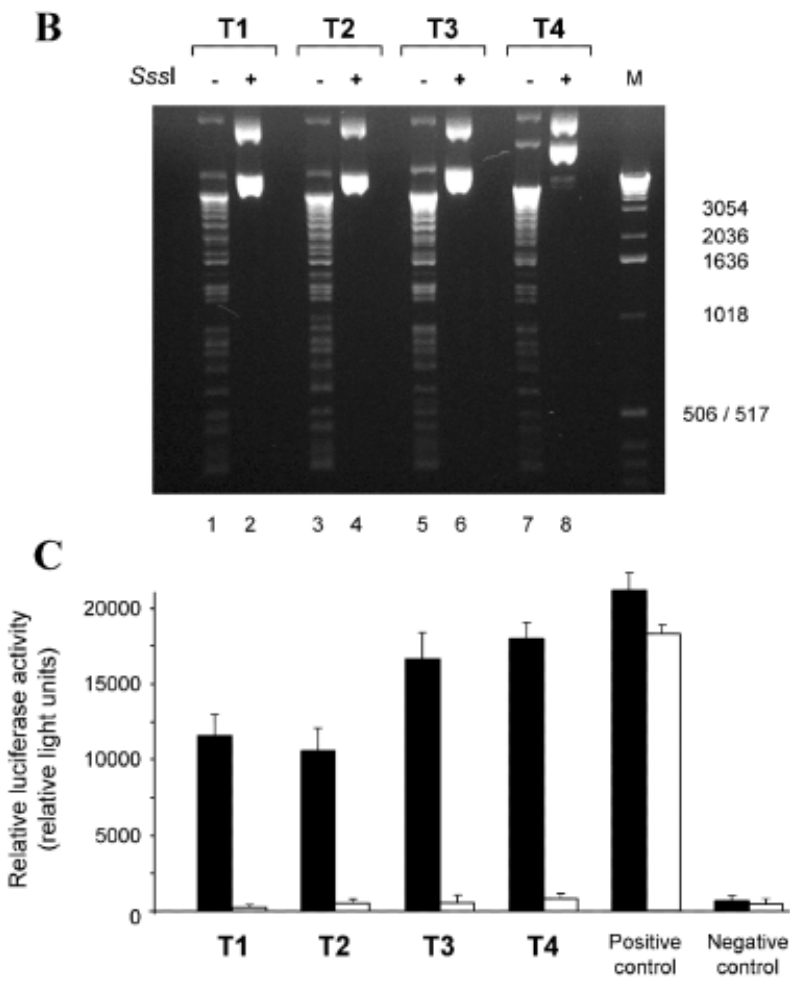

Fig. 2 Inhibition of Human TFPI-2 Promoter Activity by SssI Methyltransferase.

Various deletion constructs of the 5'-flanking region of the with $17 \%$ endotoxin-free heat-inactivated fetal calf serum (FCS, ATGC Biotechnologie, Noisy le Grand, France). The human JAR cell line was obtained from the American Type Culture Collection (Rockville, MD, USA) and grown in RPMI-1640 medium (Invitrogen, Cergy Pontoise, France) supplemented with 10\% FCS. To assess reactivation of TFPI-2 expression in JAR cells, cells were treated for $48 \mathrm{~h}$ with $1 \mu \mathrm{m}$ 5-aza (Sigma Aldrich Chimie, Saint Quentin Fallavier, France) and then for $48 \mathrm{~h}$ with freshly prepared medium containing $5 \mu \mathrm{m} 5$-aza. All cells were unstimulated (US) or stimulated with $100 \mathrm{ng} / \mathrm{ml}$ PMA for $4 \mathrm{~h}$ (PMA). Total mRNA was isolated from $2 \times 10^{5}$ trophoblast cells stimulated with PMA or left unstimulated using the Dynabeads mRNA Direct kit (Dynal France $S A$, Compiègne, France) according to the manufacturer's instructions. Total mRNA ( $40 \mathrm{ng}$ for $2 \times 10^{5}$ cells) was then reverse transcribed as previously described (lochmann et al., 2002). PCR was performed using first-strand cDNA obtained from $5 \times 10^{4}$ cells and $1 \mu \mathrm{M}$ of forward and reverse oligonucleotide primers (Genset SA, Paris, France) specific for TFPI-2 or GAPDH. PCR carried out for 35 cycles $\left(94^{\circ} \mathrm{C}\right.$ for $30 \mathrm{~s}, 62^{\circ} \mathrm{C}$ for $30 \mathrm{~s}$ and $72^{\circ} \mathrm{C}$ for $30 \mathrm{~s}$ ) and RT-PCR products were sequenced on both strands with the reverse and forward primers on a Perkin Elmer Abi Prism ${ }^{\mathrm{TM}} 377$ DNA sequencer. RT-PCR products were analyzed by electrophoresis through $1.6 \%$ agarose gels containing $1 \mu \mathrm{g} / \mathrm{ml}$ ethidium bromide. Lane M, $\phi$ X174 RF DNA/HaelII DNA ladder; *, negative control (PCR without DNA). Data are representative of at least three independent experiments.
TFPI-2 gene promoter were generated by PCR using modified primers containing restriction enzyme recognition sites (Table 1). The Xhol and Hindlll restriction enzyme-digested fragments obtained (2257, 534, 260 and 124 bp) were subsequently subcloned in the promoter-/enhancerless expression vector pGL3-Basic (Promega, Charbonnières, France) upstream from the luciferase reporter gene and all promoter constructs ( $\mathrm{T} 1, \mathrm{~T} 2$, $\mathrm{T} 3$ and $\mathrm{T} 4$, respectively) were sequenced on both strands. The TFPI-2/luciferase reporter constructs (TFPI-2/Luc) were methylated using SssI methyltransferase for $16 \mathrm{~h}$ at $37^{\circ} \mathrm{C}$ in NEBuffer 2 (New England Biolabs Ltd, Oty, UK) containing $180 \mu \mathrm{M} \mathrm{S-}$ adenosylmethionine, 10 units of Sssl methyltransferase and $5 \mu \mathrm{m}$ of plasmids. The reactions were terminated by heating for $15 \mathrm{~min}$ at $65^{\circ} \mathrm{C}$. Complete methylation of constructs was checked by evaluating their resistance to cleavage by BstUI restriction endonuclease (New England Biolabs). Only plasmids that were completely resistant to digestion were then used in the transfection experiments. (A) Schematic map of the fragments T1 to T4. (B) BstUI digestion of human TFPI-2/Luc before $(-)$ and after $(+)$ SssI treatment. Digestion products were analyzed by electrophoresis through $1.6 \%$ agarose gel containing $1 \mu \mathrm{g} / \mathrm{ml}$ ethidium bromide. Lane $\mathrm{M}, 1 \mathrm{~kb}$ ladder of DNA size marker containing DNA fragments ranging from 75 to 12216 bp. (C) The unmethylated (white) and methylated (black) human TFPI-2/Luc (1.1 nM) and plasmid controls were transiently transfected into the JAR choriocarcinoma cell line using LipofectAMINE Plus ${ }^{\mathrm{TM}}$ Reagent (Invitrogen) according to the manufacturer's instructions. The BGal plasmid (pSVbeta-galactosidase control vector, Promega) was always co-transfected to normalize transfection efficacy. Cells were harvested $24 \mathrm{~h}$ after transfection, and luciferase activity was measured using a Wallac Victor 2 multilabel reader luminometer (Perkin Elmer, Le Blanc Mesnil, France) and expressed in relative light units (RLU). A positive control containing CMV promoter sequence (pGL3 control) and a negative control (pGL3-Basic) were used for each experiment. Data are the mean \pm SEM of at least three independent transfections. 
was not detected in JAR cells, even after incubation with PMA for $4 \mathrm{~h}$. These results demonstrate a discrepancy in TFPI-2 mRNA expression between normal and tumor cells, and we hypothesized that TFPI-2 gene expression is repressed in JAR cells. We therefore analyzed the TFPI-2 gene promoter sequence and identified a complete $\mathrm{CpG}$ island region that spans exon 1 and the three transcription initiation sites. This finding suggests that the methylation status of the human TFPI-2 gene promoter could regulate the level of expression of TFPI-2 in trophoblast cells.

To demonstrate that hypermethylation of the TFPI-2 gene is responsible for the downregulation of TFPI-2 mRNA synthesis in JAR cells, we treated cells with 5-aza, a demethylating agent that has been shown to reactivate the expression of many genes in several tumor cell lines (Guillaudeux et al., 1995; Hanski et al., 1997; Christman, 2002). After treatment with 5-aza, TFPI-2 mRNA synthesis was restored in JAR choriocarcinoma cells (Figure 1B), which was probably related to the decreased methylation status of the TFPI-2 5'-flanking region. In addition, TFPI-2 mRNA expression was increased when 5-aza-treated JAR cells were incubated with PMA, whereas JAR cells without 5-aza treatment did not express TFPI-2 (Figure 1A). The DNA methylation status might affect the ability of transcription factors involved in the PMA-dependent signaling pathway to bind to their recognition sites, as previously demonstrated for AP-2, c-Myc, CREB and NF-KB (Bergman et Mostoslavsky, 1998).

The effects of in vitro DNA methylation on TFPI-2 promoter function were also confirmed with TFPI-2/Luc methylated in vitro with Sssl methyltransferase which recognizes CpG sites. Total methylation was confirmed by resistance to cleavage with BstUI restriction endonuclease. The promoter constructs used were previously described (Hubé et al., 2003b) and contained promoter sequences -2299/- 42 (T1), -576/-42 (T2), -302/-42 (T3) and $-166 /-42$ (T4) from the translation start site, comprising transcription factor binding sites necessary for transcriptional activity. Methylated or unmethylated TFPI-2/Luc plasmids were then transiently transfected into JAR cells and the luciferase activity of each promoter deletion construct (normalized with BGal values) was compared to that of the pGL3 positive control.

Figure 2A shows that no band smaller than $5 \mathrm{~kb}$ was detected in agarose gels (lanes 2, 4, 6 and 8), demonstrating the efficiency of SssI methyltransferase treatment with all plasmid constructs. Indeed, only completely methylated plasmids were resistant to digestion with methylation-sensitive BstUI restriction endonuclease and were then used in the transfection experiments.

The luciferase activity of each promoter construct was abolished after methylation with Sssl, in contrast to the activity of unmethylated constructs (Figure 2B). Moreover, hypermethylation of the T4 construct that we previously defined as the minimal promoter (Hubé et al., 2003b) greatly decreased luciferase activity. These results indicate that hypermethylation of the $\mathrm{CpG}$ island

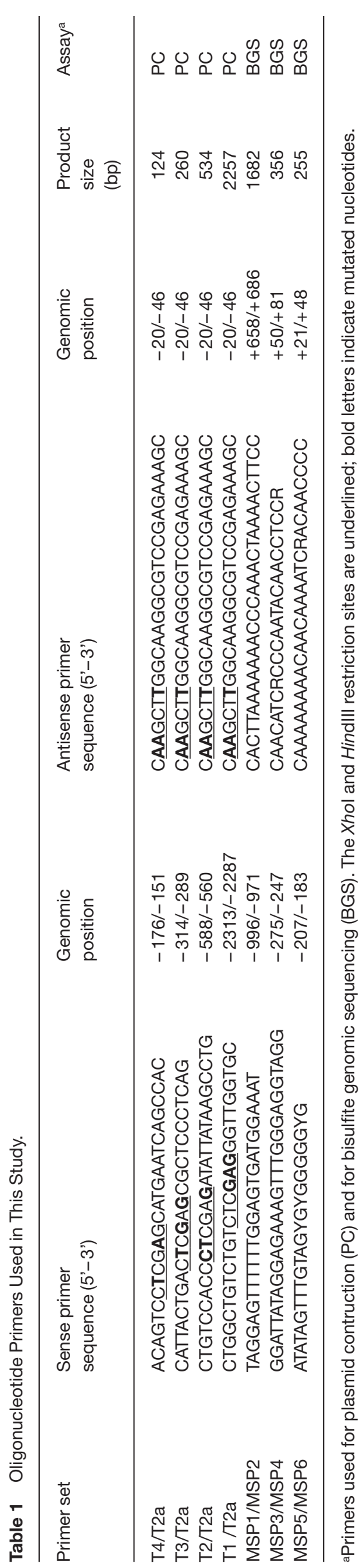


within the minimal promoter is sufficient to affect TFPI-2 expression, in agreement with previous studies that indicated a correlation between CpG methylation of the minimal promoter and transcriptional silencing (Singal et al., 1997; Kinoshita et al., 2000).

Bisulfite genomic sequencing was used to determine precisely the methylation status of each $\mathrm{CpG}$ dinucleotide within a 250 bp region of the TFPI-2 gene promoter. The sodium bisulfite treatment converts unmethylated cytosines to uracil residues, while 5-methylcytosines remain unmodified. Sequencing the PCR products of bisulfitetreated DNA allows a determination of the methylation profiles of individual DNA molecules. Twenty-eight putative CpG sites were studied in a TFPI-2 region of about 250 bp $(-207 /+48$ from the translation start site) which spanned the three transcription initiation sites and the translation start site and included the TFPI-2 minimal promoter. After amplification by nested PCR using modified primers (Table 1), PCR products were sequenced on both strands and the percentage of cytosine methylation was calculated as the number of methylated cytosines divided by the number of putative CpG dinucleotides. Figure 3 shows that $97.5 \%$ of $24 \mathrm{CpG}$ sites were methylated in JAR cells that did not express TFPI-2 mRNA. By contrast, the $\mathrm{CpG}$ island was found to be poorly methylated $(6.3 \%$ of 28 CpG's) in normal trophoblast cells that synthesized large amounts of TFPI-2 mRNA. Interestingly, methylation of the promoter region of JAR cells treated with 5-aza was $51.3 \%$, with two regions particularly hypomethylated (CpG $12-18$ and $\mathrm{CpG} 24-27)$ and two regions hypermethylated (CpG 4-11 and CpG 19-23). Significant TFPI-2 mRNA synthesis was observed in 5-aza-treated cells but remained lower than that of normal trophoblast cells. These results strongly support the hypothesis that, as previously demontrated for several other genes (Hanski et al., 1997; Esteller, 2002), hypermethylation of CpG sites of the TFPI-2 promoter is correlated with the transcriptional repression of this gene in JAR choriocarcinoma cells.

In addition, our results with JAR 5-aza cells suggest

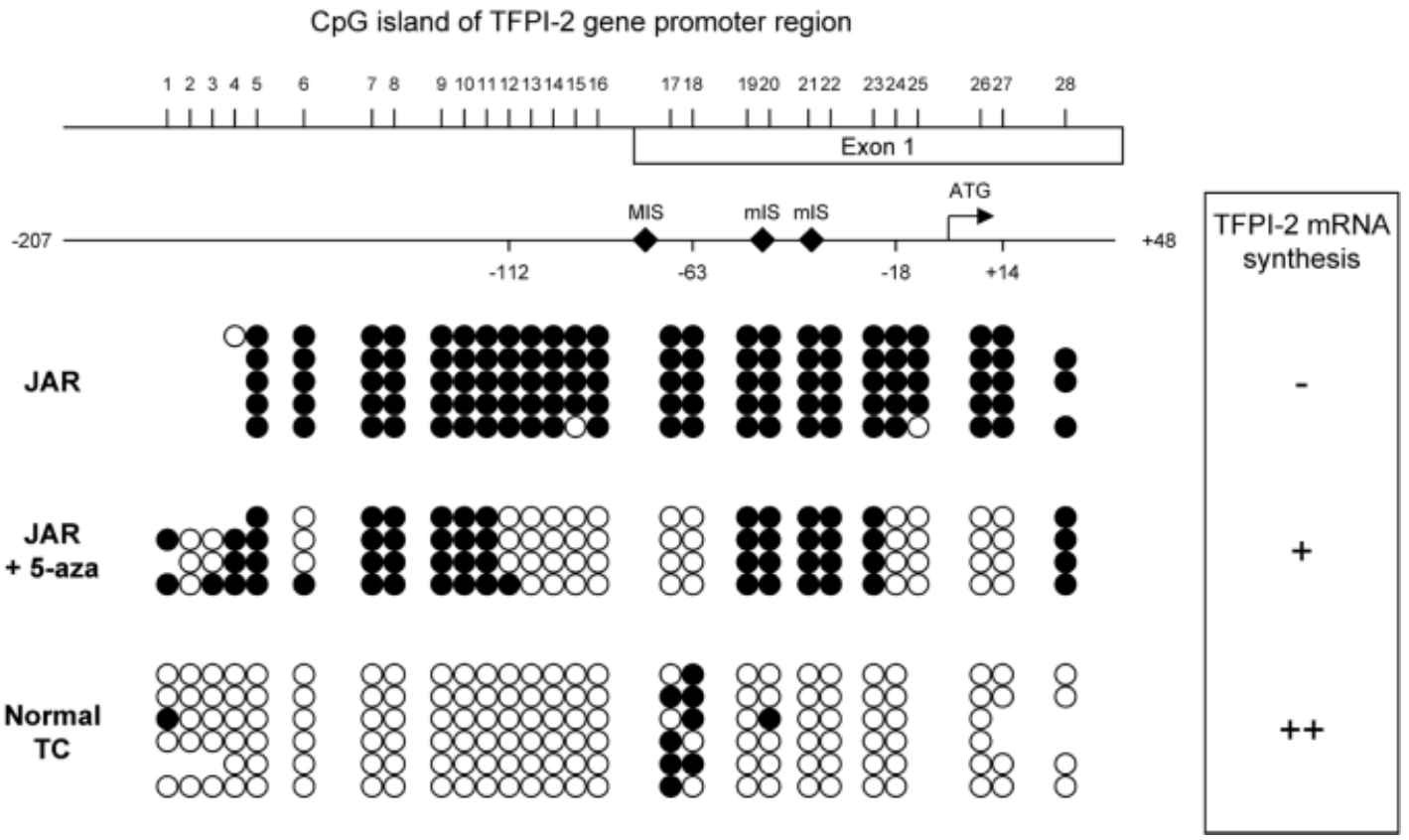

Fig. 3 Methylation Analysis of the TFPI-2 Gene Promoter.

The CpG methylation status of a $250 \mathrm{pb}$ region $(-207 /+48$ from translation start site) of the TFPI-2 promoter was studied by bisulfite genomic sequencing in untreated choriocarcima cells (JAR), JAR cells treated with 5-aza (JAR + 5-aza) and normal trophoblast cells (TC). Genomic DNA was isolated from cells using the DNeasy Tissue kit (Qiagen, Courtaboeuf, France) and converted using sodium bisulfite conversion, following the protocol of Frommer et al. (1992) with minor modifications. Briefly, $4 \mu \mathrm{g}$ of DNA were digested overnight with 5 units of HindllI. Digested DNA was purified through columns of the GFX PCR DNA and Gel Purification Kit (Amersham Pharmacia Biotech Europe GMBH, Paris, France) and then denatured in $0.3 \mathrm{M} \mathrm{NaOH}$ supplemented with $10 \mu \mathrm{g}$ tRNA (Sigma Aldrich Chimie) for $20 \mathrm{~min}$ at $42^{\circ} \mathrm{C}$. Sodium bisulfite solution generated by sodium metabisulfite (Sigma Aldrich Chimie) was added at a final concentration of $3 \mathrm{M}$, layered with mineral oil and incubated for $16 \mathrm{~h}$ at $50^{\circ} \mathrm{C}$ in the dark with $20 \mathrm{~mm}$ hydroquinone, $\mathrm{pH}$ 5.0. DNA was desalted with the GFX PCR DNA and Gel Purification Kit, desulfonated with $0.3 \mathrm{M} \mathrm{NaOH}$, and ethanol precipitated. DNA was then amplified using nested PCR. The first reaction used about $80 \mathrm{ng}$ of bisulfite-modified DNA, outer modified primers (Table 1 ) and was carried out for 35 cycles $\left(94^{\circ} \mathrm{C}\right.$ for $30 \mathrm{~s}, 60^{\circ} \mathrm{C}$ for $30 \mathrm{~s}$ and $72^{\circ} \mathrm{C}$ for $\left.60 \mathrm{~s}\right)$. The nested PCR reactions were performed using a 25 -fold dilution of PCR products with inner modified primers (Table 1). PCR products were then purified and sequenced, and the sequences were analyzed by comparing them with the original sequences. Numbers at the top (1 to 28) correspond to the relative position of $\mathrm{CpG}$ sites in this region. Major (MIS) and minor transcription initiation sites (mIS) are indicated. Each row of circles represents a single PCR product which was sequenced on both strands and each circle represents a single CpG site (open circles, non-methylated cytosines; filled circles, methylated cytosines). Results were obtained from at least three independent DNA extractions and bisulfite conversions. 
that unmethylated regions between $-112 /-63$ and - 18/ +14 (CpG 12-18 and 24-27) may allow TFPI-2 transcription initiation in this cell line. Moreover, hypermethylation of regions between $-183 /-113$ and $-54 /-20$ (CpG 4-11 and 19-23) appeared necessary but not sufficient to suppress TFPI-2 synthesis. Interestingly, the region between $-112 /-63$, which was demethylated after 5-aza treatment, contains the major transcription initiation site previously identified at position -75 upstream from the translation start site (Hubé et al., 2003b). Additional data on other tumoral cell lines will be necessary to verify the importance of these regions in TFPI-2 gene transcription.

In conclusion, the present study shows that among the multiple mechanisms that could regulate TFPI-2 expression, hypermethylation of the promoter region $\mathrm{CpG}$ island is a likely epigenetic modification. This mechanism might in choriocarcinoma contribute to a decrease in TFPI-2 synthesis, thereby favoring cancer invasion. Lakka et al. (2000) demonstrated that the downregulation of TFPI-2 with an antisense strategy increased the invasiveness of lung cancer cells. Moreover, another study showed that the transfection of JAR choriocarcinoma cells with a TFPI-2 expression vector reduced their invasive potential in vitro and in vivo (Jin et al., 2001). Further studies are necessary to verify the significance of TFPI-2 gene inactivation in choriocarcinomas from patients.

\section{Acknowledgments}

We are grateful to Prof. G. Body and Prof. F. Perrotin (Département de Gynecologie et Obstétrique, CHRU de Tours, France) who provided term placentas. We particularly thank Antoine Touzé (EMI-U0010, Tours) for the gift of pGL3 positive control. We also thank D. Raine for editing the English. This study was supported by the Ligue Contre Le Cancer and IRTH.

\section{References}

Bergman, Y., and Mostoslavsky, R. (1998). DNA demethylation: turning genes on. Biol. Chem. 379, 401-407.

Christman, J.K. (2002). 5-Azacytidine and 5-aza-2'-deoxycytidine as inhibitors of DNA methylation: mechanistic studies and their implications for cancer therapy. Oncogene 21, 5483-5495.

Esteller, M. (2002). CpG island hypermethylation and tumor suppressor genes: a booming present, a brighter future. Oncogene 21, 5427-5440.

Frommer, M., McDonald, L.E., Millar, D.S., Collis, C.M., Watt, F., Grigg, G.W., Molloy, P.L., and Paul, C.L. (1992). A genomic sequencing protocol that yields a positive display of 5-methylcytosine residues in individual DNA strands. Proc. Natl. Acad. Sci. USA 89, $1827-1831$.

Guillaudeux, T., Rodriguez, A.M., Girr, M., Mallet, V., Ellis, S.A., Sargent, I.L., Fauchet, R., Alsat, E., and Le Bouteiller, P. (1995). Methylation status and transcriptional expression of the MHC class I loci in human trophoblast cells from term placenta. J. Immunol. 154, 3283-3299.

Hanski, C., Riede, E., Gratchev, A., Foss, H.D., Bohm, C.,
Klussmann, E., Hummel, M., Mann, B., Buhr, H.J., Stein, H., Kim, Y.S., Gum, J., and Riecken, E.O. (1997). MUC2 gene suppression in human colorectal carcinomas and their metastases: in vitro evidence of the modulatory role of DNA methylation. Lab. Invest. 77, 685-695.

Herman, M.P., Sukhova, G.K., Kisiel, W., Foster, D., Kehry, M.R., Libby, P., and Schonbeck, U. (2001). Tissue factor pathway inhibitor-2 is a novel inhibitor of matrix metalloproteinases with implications for atherosclerosis. J. Clin. Invest. 107, 11171126.

Hubé, F., Reverdiau, P., lochmann, S., Trassard, S., Thibault, G., and Gruel, Y. (2003a). Demonstration of tissue factor pathway inhibitor-2 (TFPI-2) mRNA synthesis by pure villous cytotrophoblast cells isolated from term human placentas. Biol. Reprod. $68,1888-1894$.

Hubé, F., Reverdiau, P., lochmann, S., Cherpi-Antar, C., and Gruel, Y. (2003b) Characterization and functional analysis of TFPI-2 gene promoter in a human choriocarcinoma cell line. Thromb. Res. 109, 207-215.

lochmann, S., Reverdiau-Moalic, P., Hubé, F., Bardos, P., and Gruel, Y. (2002). Demonstration of inducible TFPI-2 mRNA synthesis in BeWo and JEG-3 trophoblast cells using a competitive RT-PCR. Thromb. Res. 105, 217-223.

Jin, M., Udagawa, K., Miyagi, E., Nakazawa, T., Hirahara, F., Yasumitsu, H., Miyazaki, K., Nagashima, Y., Aoki, I., and Miyagi, Y. (2001). Expression of serine proteinase inhibitor PP5/ TFPI-2/MSPI decreases the invasive potential of human choriocarcinoma cells in vitro and in vivo. Gynecol. Oncol. 83, $325-333$.

Kinoshita, H., Shi, Y., Sandefur, C., Meisner, L.F., Chang, C., Choon, A., Reznikoff, C.R., Bova, G.S., Friedl, A., and Jarrard, D.F. (2000). Methylation of the androgen receptor minimal promoter silences transcription in human prostate cancer. Cancer Res. 60, 3623-3630.

Konduri, S.D., Tasiou, A., Chandrasekar, N., Nicolson, G.L., and Rao, J.S. (2000). Role of tissue factor pathway inhibitor-2 (TFPI-2) in amelanotic melanoma (C-32) invasion. Clin. Exp. Metastasis 18, 303-308.

Konduri, S.D., Rao, C.N., Chandrasekar, N., Tasiou, A., Mohanam, S., Kin, Y., Lakka, S.S., Dinh, D., Olivero, W.C., Gujrati, M. et al. (2001). A novel function of tissue factor pathway inhibitor-2 (TFPI-2) in human glioma invasion. Oncogene 20, $6938-6945$.

Lakka, S.S., Konduri, S.D., Mohanam, S., Nicolson, G.L., and Rao, J.S. (2000). In vitro modulation of human lung cancer cell line invasiveness by antisense cDNA of tissue factor pathway inhibitor-2. Clin. Exp. Metastasis 18, 239-244.

Miyagi, Y., Yasumitsu, H., Eki, T., Miyata, S., Kkawa, N., Hirahara, F., Aoki, I., Misugi, K., and Miyazaki, K. (1996). Assignment of the human PP5/TFPI-2 gene to 7q22 by FISH and PCR-based human/rodent cell hybrid mapping panel analysis. Genomics 35, 267-268.

Rao, C.N., Cook, B., Liu, Y., Chilukuri, K., Stack, M.S., Foster, D.C., Kisiel, W., and Woodley, D.T. (1998). HT-1080 fibrosarcoma cell matrix degradation and invasion are inhibited by the matrix-associated serine protease inhibitor TFPI-2/33 kDa MSPI. Int. J. Cancer 76, 749-756.

Rao, C.N., Mohanam, S., Puppala, A., and Rao, J.S. (1999). Regulation of ProMMP-1 and ProMMP-3 activation by tissue factor pathway inhibitor-2/matrix-associated serine protease inhibitor. Biochem. Biophys. Res. Commun. 255, 94-98.

Rao, C.N., Lakka, S.S., Kin, Y., Konduri, S.D., Fuller, G.N., Mohanam, S., and Rao, J.S. (2001). Expression of tissue factor pathway inhibitor 2 inversely correlates during the progression of human gliomas. Clin. Cancer Res. 7, 570-576.

Singal, R., Ferris, R., Little, J.A., Wang, S.Z., and Ginder, G.D. 
(1997). Methylation of the minimal promoter of an embryonic globin gene silences transcription in primary erythroid cells. Proc. Natl. Acad. Sci. USA 94, 13724-13729.

Sprecher, C.A., Kisiel, W., Mathewes, S., and Foster, D.C. (1994). Molecular cloning, expression, and partial characterization of a second human tissue-factor-pathway inhibitor. Proc. Natl. Acad. Sci. USA 91, 3353-3357.

Received January 15, 2003; accepted March 3, 2003 